\title{
Shape invariance in phase space
}

\author{
Constantin Rasinariu \\ Columbia College Chicago, Department of Science and Mathematics \\ Chicago, IL 60605, Email: crasinariu@colum.edu
}

\begin{abstract}
Shape invariance is a powerful solvability condition, that allows for complete knowledge of the energy spectrum, and eigenfunctions of a system. After a short introduction into the deformation quantization formalism, this paper explores the implications of the supersymmetric quantum mechanics and shape invariance techniques to the phase space formalism. We show that shape invariance induces a new set of relations between the Wigner functions of the system, that allows for their direct calculation, once we know one of them. The simple harmonic oscillator and the Morse potential are solved as examples.
\end{abstract}

Keywords: Deformation quantization, Shape invariance, SUSYQM.

\section{INTRODUCTION}

The phase space quantum mechanics formalism (also known as deformation quantization) uses complex functions acting on the phase space instead of operators acting on a Hilbert space. These functions are endowed with a novel multiplication rule, the $*$-product, which is non-commutative, associative, and hermitian. The formalism maps operators $\hat{A}$ of quantum mechanics to complex functions $A(x, p)$, and vice versa. Historically the fourth way of doing quantum mechanics, the deformation quantization is an equivalent way of doing quantum mechanics. As Dirac noted [14]

Two points of view may be mathematically equivalent and you may think for that reason if you understand one of them you need not bother about the other and can neglect it. But it may be that one point of view may suggest a future development which another point does not suggest, and although in their present state the two points of view are equivalent they may lead to different possibilities for the future. Therefore, I think that we cannot afford to neglect any possible point of view for looking at Quantum Mechanics and in particular its relation to Classical Mechanics.

The goal of this work is to give a short introduction to the phase space quantum formalism, and to explore some of its implications as seen through the prism of the supersymmetric quantum mechanics (SUSQM). We show that shape invariance induces a new set of relations between the Wigner functions of the system, that allows for their direct calculation, once we know one of them. The paper is organized in three parts. In the first part we briefly present the main concepts of the phase space quantum mechanics formalism. In the second part we introduce SUSYQM and emphasize the role of shape invariance (SI) in the solvability of the system. In the third part we analyze some of the implications of SUSYQM and SI in deformation quantization and derive a recursion formula between the Wigner functions of the system. We calculate the simple harmonic oscillator and the Morse potential as a concrete examples.

\section{QUANTUM MECHANICS ON PHASE SPACE}

In the following, we will use the "hat" notation to symbolize quantum operators and the "non-hat" notation for the corresponding functions on the phase space. We will also consider only the one-dimensional case, where the phase space is simply $(x, p)$. For the general case, see 34 .

\section{The star product}

To any quantum operator $\hat{A}$ we associate a phase space function $A(x, p)$ using the Weyl's transform $\mathscr{W}(\hat{A})$ [34, which in the coordinate $|x\rangle$ basis, reads

$$
\mathscr{W}(\hat{A}) \equiv A(x, p)=\hbar \int d y e^{-i p y}\left\langle x+\frac{\hbar y}{2}|\hat{A}| x-\frac{\hbar y}{2}\right\rangle .
$$

All integrals run from $-\infty$ to $+\infty$, unless specifically restricted. The complex function $A(x, p)=\mathscr{W}(\hat{A})$ is also known as the Weyl's symbol of $\hat{A}$. Without other additional constraints, the reciprocal map $\mathscr{W}^{-1}$ is not unique. A sufficient condition for the unicity of $\mathscr{W}^{-1}$ is obtained by choosing an operator ordering [30. We choose Weyl's ordering, which prescribes symmetrical ordered polynomials in $\hat{x}$ and $\hat{p}$. I.e., $\mathscr{W}^{-1}(x p)=(\hat{x} \hat{p}+\hat{p} \hat{x}) / 2$. Thus, we write the reciprocal map as

$$
\begin{aligned}
\hat{A}(\hat{x}, \hat{p}) & \equiv \mathscr{W}^{-1}(A(x, p)) \\
& =\frac{1}{(2 \pi)^{2}} \int d u d v d x d p A(x, p) e^{i(\hat{p}-p) u+i(\hat{x}-x) v} .
\end{aligned}
$$

With this definition, $\mathscr{W}^{-1}(x)=\hat{x}$ and $\mathscr{W}^{-1}(p)=\hat{p}$. More generally, one can readily check that $\mathscr{W}^{-1}((a x+$ $\left.b p)^{n}\right)=(a \hat{x}+b \hat{p})^{n}$. 
Next, we define the phase space product between the Weyl's symbols. This product should correspond to the product of the quantum operators in the Hilbert space. Groenewold 21] and Moyal 28] showed that using Weyl's ordering assumption, the product of functions on phase space can be written as

$$
A(x, p) * B(x, p)=A(x, p) e^{\frac{i \hbar}{2}\left(\overleftarrow{\partial_{x}} \overrightarrow{\partial_{p}}-\overleftarrow{\partial_{p}} \overrightarrow{\partial_{x}}\right)} B(x, p),
$$

where the arrow indicates the direction in which the derivative acts. Using (1) one can prove that indeed $\mathscr{W}(\hat{A} \hat{B})=A(x, p) * B(x, p)$.

The Groenewold-Moyal product is also known as the star-product (*-product) of $A(x, p)$ and $B(x, p)$. It can be expressed in several equivalent forms, such as

$$
A(x, p) * B(x, p)=e^{\frac{i \hbar}{2}\left(\partial_{x} \partial_{p^{\prime}}-\partial_{p} \partial_{x^{\prime}}\right)} A(x, p) B\left(x^{\prime}, p^{\prime}\right),
$$

calculated at $\left(x^{\prime}, p^{\prime}\right)=(x, p)$; or, using the Bopp shifts 8]

$$
\begin{aligned}
A(x, p) * B(x, p) & =A\left(x+\frac{i \hbar}{2} \overrightarrow{\partial_{p}}, p-\frac{i \hbar}{2} \overrightarrow{\partial_{x}}\right) B(x, p)(5) \\
& =A(x, p) B\left(x-\frac{i \hbar}{2} \overleftarrow{\partial_{p}}, p+\frac{i \hbar}{2} \overleftarrow{\partial_{x}}\right)
\end{aligned}
$$

The $*$-product can also be represented as an integral, such as the Fourier representation 3, 33.

$$
\begin{aligned}
A(x, p) * B(x, p)= & \frac{1}{(\pi \hbar)^{2}} \int d x_{1} d p_{1} d x_{2} d p_{2} \\
& A\left(x_{1}, p_{1}\right) B\left(x_{2}, p_{2}\right) \\
& \times e^{-\frac{2 i}{\hbar}\left[p\left(x_{1}-x_{2}\right)+x\left(p_{2}-p_{1}\right)+\left(x_{2} p_{1}-x_{1} p_{2}\right)\right]},
\end{aligned}
$$

or as the alternate integral [23]

$$
\begin{aligned}
A(x, p) * B(x, p)= & \frac{1}{(\pi \hbar)^{2}} \int d x_{1} d p_{1} d x_{2} d p_{2} \\
& A\left(x+x_{1}, p+p_{1}\right) B\left(x+x_{2}, p+p_{2}\right) \\
& \times e^{\frac{2 i}{\hbar}\left(x_{1} p_{2}-x_{2} p_{1}\right)} .
\end{aligned}
$$

We conclude this definition by emphasizing the cyclic, trace-like properties [12] of the $*$-product:

$$
\begin{aligned}
\int d x d p A(x, p) * B(x, p) & =\int d x d p A(x, p) B(x, p) \\
& =\int d x d p B(x, p) * A(x, p \gamma(8)
\end{aligned}
$$

Thus, we have constructed a consistent way of moving back and forth from quantum operators -acting on a Hilbert space, to complex functions -acting on the phase space

$$
\begin{aligned}
\mathscr{W}(\hat{A} \hat{B}) & =\mathscr{W}(\hat{A}) * \mathscr{W}(\hat{B}), \\
\mathscr{W}^{-1}(A * B) & =\mathscr{W}^{-1}(A) \mathscr{W}^{-1}(B)
\end{aligned}
$$

This novel product is in agreement with the product rules for the operators in quantum mechanics. It is noncommutative

$$
A * B \neq B * A,
$$

associative

$$
A *(B * C)=(A * B) * C,
$$

and hermitian

$$
\overline{A * B}=\bar{B} * \bar{A},
$$

where the bar denotes the complex conjugation.

For example, using Bopp's shifts (5), we obtain:

$$
\begin{aligned}
& x * p \equiv\left(x+\frac{i \hbar}{2} \overrightarrow{\partial_{p}}\right) p=x p+\frac{i \hbar}{2} \\
& p * x \equiv\left(p-\frac{i \hbar}{2} \overrightarrow{\partial_{x}}\right) x=p x-\frac{i \hbar}{2} .
\end{aligned}
$$

Then, the *-commutator, or the Moyal bracket of $x$ and $p$ is

$$
[x, p]_{*} \equiv x * p-p * x=i \hbar,
$$

which is consistent with the canonical commutation relation $[\hat{x}, \hat{p}]=i \hbar$. We see that the Moyal bracket provides a homomorphism with commutators of operators.

\section{Moyal bracket and the correspondence principle}

Here we will briefly show that it is the Moyal bracket which provides a consistent way to quantization, and not the Poisson bracket, as conjectured by Dirac.

The $*$-product and the Moyal bracket are $\hbar$ deformations [7, 22, 24] of the usual commutative (point-wise) product of functions $A B$, and of the Poisson bracket $\{A, B\}_{P}=\partial_{x} A \partial_{p} B-\partial_{p} A \partial_{x} B$. We can write

$$
\begin{aligned}
A * B & =A B+\mathcal{O}(\hbar), \\
\frac{1}{i \hbar}[A, B]_{*} & =\{A, B\}_{P}+\mathcal{O}\left(\hbar^{2}\right) .
\end{aligned}
$$

Thus, the name deformation quantization. Property (18) implies that

$$
\lim _{\hbar \rightarrow 0} \frac{1}{i \hbar}[A, B]_{*}=\{A, B\}_{P},
$$

showing that in the classical limit one recovers the Poisson bracket.

The Moyal bracket obeys Jacobi's identity

$$
\left[[A, B]_{*}, C\right]_{*}+\left[[C, A]_{*}, B\right]_{*}+\left[[B, C]_{*}, A\right]_{*}=0,
$$

as well as the Leibniz rule

$$
[A, B * C]_{*}=[A, B]_{*} * C+B *[A, C]_{*} .
$$


Thus, one can endow the space of Weyl's symbols not only with a Lie algebra structure with respect to the Moyal bracket, but also with an inner derivative. An analogous statement holds true for the commutator algebra of quantum operators, but not for the Poissonbracket algebra. Groenewold and van Hove showed [21. 31] that there is no invertible linear map from all functions of phase space $A(x, p), B(x, p), \ldots$ to Hermitian operators in Hilbert space $\hat{A}, \hat{B}, \ldots$ that will preserve the Poisson bracket structure, i.e. $\mathscr{W}^{-1}\left(\{A, B\}_{P}\right)=$ $\frac{1}{i \hbar}\left[\mathscr{W}^{-1}(A), \mathscr{W}^{-1}(B)\right]$. Using Poisson's bracket as a starting point for quantization works only in the special cases where the series (17) terminates after the first order in $\hbar$. This is true for functions at most quadratic in $p$ and $x$. As counterexample, Groenewold 21] showed that the identically zero expression in Poisson brackets

$$
\left\{x^{3}, p^{3}\right\}_{P}+3\left\{x p^{2}, x^{2} p\right\}_{P}=0
$$

in Dirac's quantization heuristics, becomes

$$
\frac{1}{i \hbar}\left[\hat{x}^{3}, \hat{p}^{3}\right]+\frac{3}{i \hbar}\left[\frac{\hat{x} \hat{p}^{2}+\hat{p}^{2} \hat{x}}{2}, \frac{\hat{x}^{2} \hat{p}+\hat{p} \hat{x}^{2}}{2}\right]=-3 \hbar^{2},
$$

thus exhibiting a deficiency of order $\hbar^{2}$.

Through the Weyl's invertible correspondence map (1), the Hilbert space of Hermitian operators endowed with the operator commutator, has as counterpart the algebra of Weyl's symbols endowed with the Moyal's bracket. Up to an isomorphism, the Lie algebra generated by the Moyal's bracket is the unique associative one-parameter deformation of the Poisson bracket [2, 5, 13, 16, 20, 32. This uniqueness is extended (up to an isomorphism) to the $*$-product. Thus, it is the Moyal's bracket that gives the correct correspondence principle for the quantization scheme [17, 21, 38].

\section{The Wigner function}

Now that we have a coherent way of modeling quantum operators and their products, we turn our attention to modeling the quantum state. A pure state is described by the ket vector $|\psi\rangle$, or equivalently, by the corresponding density operator $\hat{\rho}=|\psi\rangle\langle\psi|$. We will consider here only pure states, because the generalization to mixed states is straightforward. Let us denote by $P(x, p)$ the normalized Weyl's symbol of the density operator $\hat{\rho}$. We have

$$
\begin{aligned}
P(x, p) & \equiv \frac{1}{2 \pi \hbar} \mathscr{W}(\hat{\rho}) \\
& =\frac{1}{2 \pi} \int d y e^{-i p y}\left\langle x+\frac{\hbar y}{2} \mid \psi\right\rangle\left\langle\psi \mid x-\frac{\hbar y}{2}\right\rangle,
\end{aligned}
$$

or

$$
P(x, p)=\frac{1}{2 \pi} \int d y e^{-i p y} \psi\left(x-\frac{\hbar y}{2}\right) \bar{\psi}\left(x+\frac{\hbar y}{2}\right) .
$$

$P(x, p)$ is the celebrated Wigner function [35], and will play a central role in the deformation quantization technique. Here are several essential properties of the Wigner function 23, 25]

(i) $P(x, p)$ is real.

(ii)

$$
\begin{aligned}
& \int d p P(x, p)=|\psi(x)|^{2}=\langle x|\hat{\rho}| x\rangle \\
& \int d x P(x, p)=|\psi(p)|^{2}=\langle p|\hat{\rho}| p\rangle \\
& \int d x d p P(x, p)=1 .
\end{aligned}
$$

(iii) If $P_{\psi}(x, p)$ and $P_{\phi}(x, p)$ correspond to to the states $\psi(x)$ and $\phi(x)$ respectively, then

$$
\left|\int d x \bar{\psi}(x) \phi(x)\right|^{2}=2 \pi \hbar \int d x d p P_{\psi}(x, p) P_{\phi}(x, p) \text {. }
$$

The last property has two interesting consequences. If $\psi(x)=\phi(x)$ then

$$
\int d x d p P_{\psi}^{2}(x, p)=\frac{1}{2 \pi \hbar},
$$

and, if we choose $\psi(x)$ orthogonal to $\phi(x)$, we get

$$
\int d x d p P_{\psi}(x, p) P_{\phi}(x, p)=0 .
$$

Equation 31 implies that $P(x, p)$ cannot be everywhere positive. Because it can also take negative values, the Wigner function is also known as a pseudo-distribution.

(iv) $P(x, p)$ is the only pseudo-distribution for which each Galilei transformation corresponds to the same Galilei transformation of the quantum mechanical wave function [36]. I.e., if $\psi(x) \mapsto \psi(x+$ $a)$, then $P(x, p) \mapsto P(x+a, p)$, and if $\psi(x) \mapsto$ $\exp \left(i p^{\prime} x / \hbar\right) \psi(x)$, then $P(x, p) \mapsto P\left(x, p-p^{\prime}\right)$.

(v) If $\psi(x) \mapsto \psi(-x)$, then $P(x, p) \mapsto P(-x,-p)$, and if $\psi(x) \mapsto \bar{\psi}(x)$ then $P(x, p) \mapsto P(x,-p)$.

To describe the time evolution of the system in the phase space, let us take the time derivative of (24), and use Schrödinger's equation together with its conjugate. We get

$$
\begin{aligned}
\frac{\partial}{\partial t} P(x, p) & =\frac{1}{2 \pi i \hbar} \int d y e^{-i p y}\left\langle x+\frac{\hbar y}{2}|(\hat{H} \hat{\rho}-\hat{\rho} \hat{H})| x-\frac{\hbar y}{2}\right\rangle \\
& =\frac{1}{i \hbar} \mathscr{W}\left(\left[\hat{H}, \frac{\hat{\rho}}{2 \pi \hbar}\right]\right)
\end{aligned}
$$

or

$$
i \hbar \frac{\partial}{\partial t} P(x, p)=[H(x, p), P(x, p)]_{*},
$$


where $H(x, p)$ is the Weyl's symbol of the Hamiltonian $\hat{H}$. Equation 32 is mirroring the time evolution of the density operator $i \hbar \frac{\partial}{\partial t} \hat{\rho}=[\hat{H}, \hat{\rho}]$. The time evolution of the Wigner function can also be symbolically written using the sine notation [4, which emphasizes the nonlinear deformation involved by the Moyal bracket

$\frac{\partial}{\partial t} P(x, p)=\frac{2}{\hbar} \sin \left\{\frac{\hbar}{2}\left(\frac{\partial}{\partial x} \frac{\partial}{\partial p_{1}}-\frac{\partial}{\partial x_{1}} \frac{\partial}{\partial p}\right)\right\} H(x, p) P\left(x_{1}, p\right.$

calculated at $\left(x_{1}, p_{1}\right)=(x, p)$.

For stationary states $\frac{\partial}{\partial t} P(x, p)=0$, hence the Hamiltonian $*$-commutes with the Wigner function

$$
[H(x, p), P(x, p)]_{*}=0 \text {. }
$$

In the stationary case one can get more constraints on the Wigner function. It we apply the Weyl's transform on $\hat{H} \hat{\rho}=E \hat{\rho}$, we obtain the $*$-eignevalue equation [11, 15]

$$
H(x, p) * P(x, p)=E P(x, p),
$$

where $E$ is the energy of the system. Hermiticiy implies that $\hat{\rho} \hat{H}=E \hat{\rho}$, hence the symmetrical relation holds true as well

$$
P(x, p) * H(x, p)=E P(x, p) .
$$

Note that if $P_{E}$ and $P_{E^{\prime}}$ correspond to the eigenenergies $E$ and $E^{\prime}$, then due to the associativity of the *-product, we have

$$
P_{E} * H * P_{E^{\prime}}=E P_{E} * P_{E^{\prime}}=E^{\prime} P_{E} * P_{E^{\prime}} .
$$

If $E \neq E^{\prime}$, it follows that $P_{E} * P_{E^{\prime}}=0$. In the general case we have $P_{E} * P_{E^{\prime}}=\mathscr{W}\left(\hat{\rho}_{E} \hat{\rho}_{E^{\prime}}\right) /(2 \pi \hbar)^{2}$ which yields the orthogonality-idempotence relation of Wigner functions

$$
P_{E} * P_{E^{\prime}}=\frac{\delta_{E, E^{\prime}}}{2 \pi \hbar} P_{E}
$$

In deformation quantization, $P(x, p)$ plays an analogous role to the probability density function in classical statistical mechanics. Namely, the average of $\hat{A}$ in state $|\psi\rangle$ is given by 28

$$
\langle\psi|\hat{A}| \psi\rangle \equiv\langle A(x, p)\rangle=\int d x d p P(x, p) * A(x, p) .
$$

Note the resemblance with statistical mechanics. However there is a major difference in the case of the deformation quantization formalism: the function $P(x, p)$ is not a probability distribution in the statistical sense, because it can take negative values.

\section{The harmonic oscillator}

To exemplify the concepts introduced so far, let us consider the case of the simple harmonic oscillator. Without loss of generality we take $2 m=1, \omega=2$. Then the Hamiltonian operator of the harmonic oscillator reads

$$
\hat{H}=\hat{p}^{2}+\hat{x}^{2},
$$

having the corresponding Weyl symbol

$$
H(x, p)=p^{2}+x^{2} .
$$

$p_{\text {击 }}$ et us write the $*$-eigenvalue equation 34 for this case. We obtain

$$
\left(p^{2}+x^{2}\right) * P(x, p)=E P(x, p),
$$

which, using Bopp's shifts representation (5) becomes

$$
\left[\left(p-\frac{i \hbar}{2} \overrightarrow{\partial_{x}}\right)^{2}+\left(x+\frac{i \hbar}{2} \overrightarrow{\partial_{p}}\right)^{2}\right] P(x, p)=E P(x, p) .
$$

Performing the algebra we arrive to

$$
\left[p^{2}+x^{2}+i \hbar\left(x \partial_{p}-p \partial_{x}\right)-\frac{\hbar^{2}}{4}\left(\partial_{p}^{2}+\partial_{x}^{2}\right)-E\right] P(x, p)=0 .
$$

After separating the imaginary and the real parts, we get

$$
\begin{aligned}
& \left(x \partial_{p}-p \partial_{x}\right) P(x, p)=0 \\
& {\left[p^{2}+x^{2}-\frac{\hbar^{2}}{4}\left(\partial_{p}^{2}+\partial_{x}^{2}\right)-E\right] P(x, p)=0 .}
\end{aligned}
$$

The symmetry of equation 43 indicates that $P(x, p)$ depends effectively of only one variable, which is a symmetric combination of $x$ and $p$. Thus, in (44) we make the change of variables $t=2\left(p^{2}+x^{2}\right) / \hbar$ and write $P(t)=e^{-t / 2} L(t)$. We get

$$
\left[t \partial_{t}^{2}+(1-t) \partial_{t}+\left(\frac{E}{2 \hbar}-\frac{1}{2}\right)\right] L(t)=0
$$

For $L(t)$ to be normalizable, the zero derivative term of (45) must be a positive integer

$$
\left(\frac{E}{2 \hbar}-\frac{1}{2}\right)=n, \quad n=0,1,2, \ldots
$$

This assures that the series solution of $L(t)$ terminates at a given rank, hence $L(t)$ is finite and $e^{-t / 2} L(t)$ is normalizable. But condition (46) yields exactly the quantization formula for the energy of the harmonic oscillator $(\omega=2)$

$$
E_{n}=2 \hbar\left(n+\frac{1}{2}\right), \quad n=0,1,2, \ldots
$$

Consequently, equation 45 subject to the constraint 46, becomes the differential equation of Laguerre polynomials $L_{n}(t)$ 9]. Thus, we obtain the analytic expression for the Wigner functions $P_{n}(x, p)$ of the harmonic oscillator

$P_{n}(x, p)=\frac{(-1)^{n}}{\pi} e^{-\frac{p^{2}+x^{2}}{\hbar}} L_{n}\left(\frac{p^{2}+x^{2}}{\hbar / 2}\right), n=0,1,2, \ldots$ 


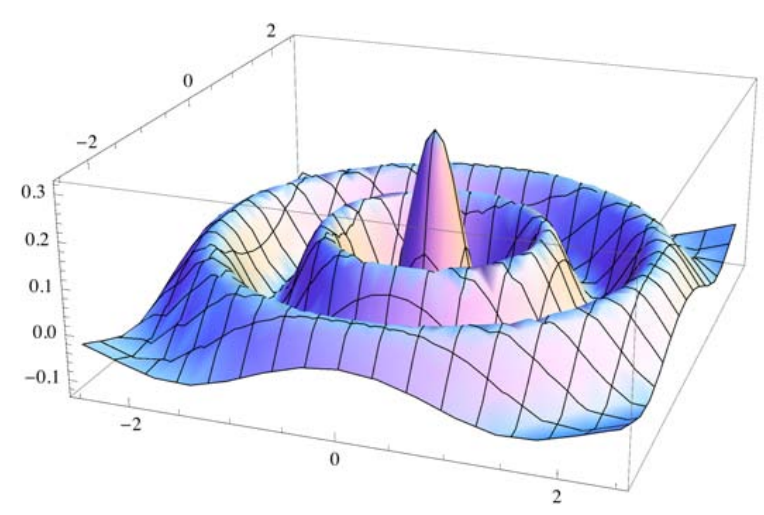

Figure 1: The Wigner function $P_{5}(x, p)$ for the simple harmonic oscillator.

where $L_{n}$ is the $n$-th Laguerre polynomial. The harmonic oscillator is one of the few systems where the Wigner functions are completely known analytically [1]. In figure (1) we illustrate the Wigner function for $n=5, m=1 / 2$, and $\omega=2$. Note the circular $x \leftrightarrow p$ symmetry of the solution, as reflected by the equation (43).

Finally, let's analyze the algebraic solution induced by the factorization of the Hamiltonian in terms of creation and annihilation operators $\hat{a}^{+}$and $\hat{a}$. This method will naturally segue into the factorization of a general Hamiltonian, which is the crux of the SUSYQM techniques. We write the Hamiltonian as

$$
\hat{H}=2 \hbar\left(\hat{a}^{+} \hat{a}+1 / 2\right),
$$

where $\hat{a}=(i \hat{p}+\hat{x}) / \sqrt{2 \hbar}$ and $\hat{a}^{+} \equiv(\hat{a})^{\dagger}=(-i \hat{p}+\hat{x}) / \sqrt{2 \hbar}$. Then $\left[\hat{a}, \hat{a}^{+}\right]=1$, and after algebraic manipulations we obtain

$$
\begin{aligned}
\hat{H}|n\rangle & =2 \hbar(n+1 / 2)|n\rangle, \quad n=0,1,2, \ldots \\
\hat{a}^{+}|n\rangle & =\sqrt{n+1}|n+1\rangle \\
\hat{a}|n\rangle & =\sqrt{n}|n-1\rangle .
\end{aligned}
$$

The harmonic oscillator wave functions are obtained by projecting the recursion relations

$$
|n\rangle=\frac{\left(\hat{a}^{+}\right)^{n}}{\sqrt{n !}}|0\rangle,
$$

onto the $|x\rangle$ basis, where the starting point is given by $\hat{a}|0\rangle=0$.

In the phase space picture these relations become

$$
H(x, p)=p^{2}+x^{2}=2 \hbar\left(a^{+} * a+1 / 2\right),
$$

where the annihilation and creation functions $a(x, p)$ and $a^{+}(x, p)$ are

$$
a=(i p+x) / \sqrt{2 \hbar} \quad, \quad a^{+} \equiv \overline{(a)}=(-i p+x) / \sqrt{2 \hbar} .
$$

A simple calculation shows that their Moyal bracket gives

$$
\left[a, a^{+}\right]_{*}=1 .
$$

Then, the $*$-eignevalue problem becomes

$$
H(x, p) * P_{n}(x, p)=2 \hbar(n+1 / 2) P_{n}(x, p),
$$

where the Wigner function $P_{n}(x, p)$ is determined from the recursion relations 12 ]

$$
P_{n}(x, p)=\frac{1}{n !}\left(a^{+} *\right)^{n} P_{0}(x, p)(* a)^{n},
$$

using as starting point

$$
a * P_{0}(x, p)=0 .
$$

A direct calculation [12] shows that the algebraic method recovers the known form for the Wigner function (48). This algebraic technique will be extended to a general Hamiltonian, by applying SUSYQM concepts to the phase space formalism.

\section{SUPERSYMMETRIC QUANTUM MECHANICS}

In this section we give a brief introduction to supersymmetric quantum mechanics (SUSYQM) techniques which we will use mostly as reference for the next section. For more details see [10, 18, 19, 37. SUSYQM provides an elegant and useful prescription for obtaining closed analytic expressions both for the energy eigenvalues and eigenfunctions for a large class of one dimensional potentials.

Given the Hamiltonian $\hat{H}=\frac{\hat{p}^{2}}{2 m}+V(\hat{x})$ with the eigenenergies $E_{n}$, let us shift its energy spectrum by $E_{0}$, such that the new ground state energy becomes zero, and denote the new Hamiltonian by $\hat{H}_{-}$, and its eigenenergies by $E_{n}^{-}$:

$$
\hat{H}_{-}=\frac{\hat{p}^{2}}{2 m}+V_{-}(\hat{x}) ; \quad \hat{H}_{-}|n ;-\rangle=E_{n}^{-}|n ;-\rangle, \quad E_{0}^{-}=0 .
$$

$\hat{H}_{-}$can be factorized [29] as

$$
\hat{H}_{-}=\hat{A}^{+} \hat{A},
$$

where

$$
\hat{A}=\frac{i \hat{p}}{\sqrt{2 m}}+W(\hat{x}), \quad \hat{A}^{+} \equiv(\hat{A})^{\dagger}=\frac{-i \hat{p}}{\sqrt{2 m}}+W(\hat{x}) .
$$

The function $\hat{W}$ is known as the superpotential and is related to $V_{-}(\hat{x})$ by

$$
V_{-}(\hat{x})=W^{2}(\hat{x})-\frac{\hbar}{\sqrt{2 m}} W^{\prime}(\hat{x}) .
$$


Equation 63 becomes a Riccati equation when projected on the $|x\rangle$ basis, thus allowing for finding $W(x)$. A sufficient condition for $E_{0}^{-}=0$ is given by

$$
\hat{A}|0 ;-\rangle=0,
$$

which yields the explicit action of $W(\hat{x})$ on the ground state eigenvector $|0 ;-\rangle$

$$
W(\hat{x})|0 ;-\rangle=-\frac{i \hat{p}}{\sqrt{2 m}}|0 ;-\rangle .
$$

If we project 65 on the $|x\rangle$ basis, we can express $W(x)$ in terms of the ground state eigenfunction $\psi_{0}^{-}(x)=\langle x \mid 0 ;-\rangle$ as 19

$$
W(x)=-\frac{\hbar}{\sqrt{2 m}} \frac{\psi_{0}^{-\prime}(x)}{\psi_{0}^{-}(x)} .
$$

By interchanging the operators $\hat{A}^{+}$and $\hat{A}$ we generate a new Hamiltonian $\hat{H}_{+}=\hat{A} \hat{A}^{+}$, which corresponds to a new potential $V_{+}(\hat{x})$

$$
\hat{H}_{+}=\frac{\hat{p}^{2}}{2 m}+V_{+}(\hat{x}) ; \quad V_{+}(\hat{x}) \equiv W^{2}(\hat{x})+\frac{\hbar}{\sqrt{2 m}} W^{\prime}(\hat{x}) .
$$

Let us denote by $E_{n}^{+}$its eigenenergies: $\hat{H}_{+}|n ;+\rangle=$ $E_{n}^{+}|n ;+\rangle$. The two Hamiltonians $\hat{H}_{-}$and $\hat{H}_{+}$are known as supersymmetric partner Hamiltonians. Their eigenvectors and eigenenergies are related

$$
\begin{aligned}
E_{n}^{+} & =E_{n+1}^{-} ; \quad E_{0}^{-}=0, \\
|n ;+\rangle & =\frac{1}{\sqrt{E_{n+1}^{-}}} \hat{A}|n+1 ;-\rangle, \\
|n+1 ;-\rangle & =\frac{1}{\sqrt{E_{n}^{+}}} \hat{A}^{+}|n ;+\rangle .
\end{aligned}
$$

Thus, with the exception of the ground state, the supersymmetric partner potentials $\hat{H}_{-}$and $\hat{H}_{+}$share the same energy spectrum (isospectrality), and have interconnected eigenvector sets. Formulas 68-70) together with (64) represent a quick algebraic way of completely finding the spectrum and eigenvectors of $\hat{H}_{+}$if we know the spectrum and eigenvectors of $\hat{H}_{-}$. In this way, if $\hat{H}_{-}$ corresponds to a very simple simple system, we have an elegant solution for solving more complicated cases associated to $\hat{H}_{+}$.

There is a special class of partner Hamiltonians for which potentials $V_{-}(\hat{x})$ and $V_{+}(\hat{x})$ obey an additional constraint known as shape invariance. Shape invariance states that partner potentials are similar in shape

$$
V_{+}\left(\hat{x} ; a_{0}\right)=V_{-}\left(\hat{x} ; a_{1}\right)+R\left(a_{0}\right),
$$

differing only by a set of parameters (modeling the strength of the interaction) $a_{0}, a_{1}=f\left(a_{0}\right)$, and a constant term $R\left(a_{0}\right)$, independent of $\hat{x}$. Shape invariance is a solvability condition that allows in principle to completely solve the system, as it will be shown shortly. For this, let us rewrite the shape invariance condition as

$$
H_{+}\left(\hat{x}, \hat{p} ; a_{0}\right)+g\left(a_{0}\right)=H_{-}\left(\hat{x}, \hat{p} ; a_{1}\right)+g\left(a_{1}\right),
$$

where $R\left(a_{0}\right)=g\left(a_{1}\right)-g\left(a_{0}\right)$. Note that we do not have to know the spectrum of one partner Hamiltonian to know the other. Since Hamiltonians in 72 differ by a constant, their eigenvalues differ by the same constant, and, up to a global phase factor, both have the same set of eigenvectors. Therefore, for all values of $n$ we have

$$
\begin{aligned}
E_{n}^{+}\left(a_{0}\right) & =E_{n}^{-}\left(a_{1}\right)+g\left(a_{1}\right)-g\left(a_{0}\right), \\
\left|n, a_{0} ;+\right\rangle & =\left|n, a_{1} ;-\right\rangle .
\end{aligned}
$$

For normalizable ground states (unbroken SUSY), the ground state energy of $H_{-}\left(\hat{x}, \hat{p} ; a_{i}\right)$ is zero, $E_{0}^{-}\left(a_{i}\right)=0$, for each iteration of the parameter $a_{i}=f\left(a_{i-1}\right)$. By successively using the shape invariance $(73)$ and the isospectrality 68 of the partner Hamiltonians, in conjunction with the unbroken supersymmetry condition, we get

$$
\begin{aligned}
E_{0}^{-}\left(a_{0}\right) & =0, \\
E_{1}^{-}\left(a_{0}\right) & =E_{0}^{+}\left(a_{0}\right) \quad \text { (isospectrality) } \\
& =E_{0}^{-}\left(a_{1}\right)+g\left(a_{1}\right)-g\left(a_{0}\right) \quad \text { (shape invariance) } \\
& =g\left(a_{1}\right)-g\left(a_{0}\right) \quad \text { (unbroken supersymmetry) } \\
& \cdots \\
E_{n}^{-}\left(a_{0}\right) & =g\left(a_{n}\right)-g\left(a_{0}\right) .
\end{aligned}
$$

Similarly,

$$
\left|n, a_{0} ;-\right\rangle=\frac{\hat{A}^{+}\left(a_{0}\right) \cdots \hat{A}^{+}\left(a_{n-1}\right)\left|0, a_{n} ;-\right\rangle}{\left[\prod_{j=0}^{n-1} E_{n-j}^{-}\left(a_{j}\right)\right]^{1 / 2}} .
$$

All eigenenergies and eigenvectors of the Hamiltonian $\hat{H}_{-}$can be determined iteratively by this algorithm. Thus, SUSYQM and shape invariance determine the entire spectrum of the system without any need to solve complicated differential equations. For more details see [18] and the references therein.

We are now ready to apply these ideas to the phase space formalism.

\section{SUSYQM AND SHAPE INVARIANCE IN PHASE SPACE}

SUSYQM on the phase space is obtained by applying Weyl's map to the operatorial framework discussed above. Thus, the Weyl symbols of the partner supersymmetric Hamiltonians $\hat{H}_{-}$and $\hat{H}_{+}$are

$$
\begin{aligned}
& H_{-}(x, p)=\frac{p^{2}}{2 m}+V_{-}(x) \equiv A^{+}(x, p) * A(x, p), \\
& H_{+}(x, p)=\frac{p^{2}}{2 m}+V_{+}(x) \equiv A(x, p) * A^{+}(x, p),
\end{aligned}
$$


where the annihilation and creation functions are given by

$$
\begin{aligned}
A(x, p) & =\frac{i p}{2 m}+W(x) \\
A^{+}(x, p) & \equiv \bar{A}(x, p)=-\frac{i p}{2 m}+W(x) .
\end{aligned}
$$

The supersymmetric partner potentials are simply

$$
V_{\mp}(x)=W^{2}(x) \mp \frac{\hbar}{\sqrt{2 m}} W^{\prime}(x) .
$$

The last formula can be obtained either by Weyl's mapping the quantum operators $V_{\mp}(\hat{x})$, or by direct calculations from (77) and (78) using the definitions 79. 80 , and the property of Moyal's bracket $[W(x), p]_{*}=$ $i \hbar \partial_{x} W(x)$.

The $*$-eigenvalue problem for the supersymmetric partner potentials $H_{-}(x, p)$ and $H_{+}(x, p)$ written in terms of their corresponding Wigner functions reads

$$
\begin{aligned}
& H_{-}(x, p) * P_{n}^{-}(x, p)=E_{n}^{-} P_{n}^{-}(x, p), \\
& H_{+}(x, p) * P_{n}^{+}(x, p)=E_{n}^{+} P_{n}^{+}(x, p) .
\end{aligned}
$$

where

$$
P_{n}^{\mp}(x, p)=\frac{1}{2 \pi} \int d y e^{-i p y}\left\langle x+\frac{\hbar y}{2} \mid n ; \mp\right\rangle\left\langle n ; \mp \mid x-\frac{\hbar y}{2}\right\rangle
$$

are the $n$-th excited state Wigner functions of the supersymmetric partner Hamiltonians $H_{\mp}(x, p)$. The necessary condition for the ground state energy $E_{0}^{-}$of $H_{-}(x, p)$ to be zero, is

$$
A(x, p) * P_{0}^{-}(x, p)=0 .
$$

This yields the $*$-product equation

$$
W(x) * P_{0}^{-}(x, p)=-\frac{i}{\sqrt{2 m}} p * P_{0}^{-}(x, p),
$$

which in terms of Bopp's shifts becomes

$$
W\left(x+\frac{i \hbar}{2} \overrightarrow{\partial_{p}}\right) P_{0}^{-}(x, p)=\frac{-i}{\sqrt{2 m}}\left(p-\frac{i \hbar}{2} \overrightarrow{\partial_{x}}\right) P_{0}^{-}(x, p) .
$$

By applying the Weyl map on equations 69 and (70) we obtain [11] the connections between the *eigenfunctions of the supersymmetric partner potentials $H_{-}(x, p)$ and $H_{+}(x, p)$

$$
\begin{aligned}
P_{n}^{+}(x, p) & =\frac{1}{E_{n+1}^{-}} A(x, p) * P_{n+1}^{-}(x, p) * A^{+}(x, p), \\
P_{n+1}^{-}(x, p) & =\frac{1}{E_{n}^{+}} A^{+}(x, p) * P_{n}^{+}(x, p) * A(x, p) .
\end{aligned}
$$

The only constraint we imposed so far was that the Hamiltonians $H_{\mp}(x, p)$ are supersymmetric partners. This leads, besides the isospectrality, to the *product connection between the corresponding Wigner functions $P_{n}^{\mp}(x, p)$. It is almost the "carbon copy" of the SUSYQM case. Next, we explore the implications of the shape invariance. In phase space, equation 72 becomes

$$
H_{+}\left(x, p ; a_{0}\right)+g\left(a_{0}\right)=H_{-}\left(x, p ; a_{1}\right)+g\left(a_{1}\right),
$$

where $a_{0}$ and $a_{1}=f\left(a_{0}\right)$. Because the Hamiltonians in (90) differ by a constant, they have the same set of *-eigenfunctions. Hence, up to a sign factor (because Wigner functions are real), shape invariance implies

$$
P_{n}^{+}\left(x, p ; a_{0}\right)=P_{n}^{-}\left(x, p ; a_{1}\right)
$$

and the energy spectrum

$$
E_{n}^{-}\left(a_{0}\right)=g\left(a_{n}\right)-g\left(a_{0}\right), \quad \text { with } a_{n}=f^{n}\left(a_{0}\right) .
$$

In addition, the shape invariance together with the supersymmetric condition, leads to a new recursion formula among the Wigner functions:

$$
P_{n}^{-}\left(x, p ; a_{0}\right)=A^{+}\left(x, p ; a_{0}\right) * \frac{P_{n-1}^{-}\left(x, p ; a_{1}\right)}{E_{n}^{-}\left(a_{0}\right)} * A\left(x, p ; a_{0}\right) .
$$

The proof is immediate:

$$
\begin{aligned}
P_{n}^{-}\left(x, p ; a_{0}\right) & =A^{+}\left(x, p ; a_{0}\right) * \frac{P_{n-1}^{+}\left(x, p ; a_{0}\right)}{E_{n-1}^{+}\left(a_{0}\right)} * A\left(x, p ; a_{0}\right), \\
& =A^{+}\left(x, p ; a_{0}\right) * \frac{P_{n-1}^{-}\left(x, p ; a_{1}\right)}{E_{n}^{-}\left(a_{0}\right)} * A\left(x, p ; a_{0}\right),
\end{aligned}
$$

where the first equality follows from SUSYQM, and the second one from shape invariance and isospectrality. We can now iterate 93 to obtain the expression for a general $P_{n}^{-}\left(x, p ; a_{0}\right)$ starting from the ground state Wigner function $P_{0}^{-}\left(x, p ; a_{n}\right)$. We have

$$
\begin{aligned}
P_{n}^{-}\left(x, p ; a_{0}\right)= & A^{+}\left(x, p ; a_{0}\right) * \cdots * A^{+}\left(x, p ; a_{n-1}\right) \\
& * \frac{P_{0}^{-}\left(x, p ; a_{n}\right)}{\prod_{j=0}^{n-1} E_{n-j}^{-}\left(a_{j}\right)} * A\left(x, p ; a_{n-1}\right) \\
& * \cdots * A\left(x, p ; a_{0}\right) .
\end{aligned}
$$

This recursion formula together with 85 as its starting point, determines, the entire set of Wigner functions for shape invariant systems. However, for concrete examples, the multiple $*$-products can become prohibitively difficult to calculate. The harmonic oscillator is one a the few cases where we can determine analytically the entire sequence of Wigner functions.

\section{The Morse potential}

As an example, let us consider the one dimensional Morse potential. The Morse oscillator is a good approximation of the oscillatory motion of the bi-atomic 
molecules [6, 27]. Without loss of generality, we take $\hbar=1$ and $2 m=1$. Then, the Hamiltonian reads

$$
H_{-}(x, p ; a)=p^{2}+V_{-}(x, a),
$$

where the Morse potential

$V_{-}(x, a)=a^{2}+b^{2} e^{-2 s x}-2 b(a+1 / 2) e^{-s x} ; \quad a, b, s>0$,

can be generated as $V_{-}=W^{2}-W^{\prime}$ from the superpotential [10]

$$
W(x ; a)=a-b e^{-s x} .
$$

The corresponding annihilation and creation phase space functions are

$$
A(x, p)=i p+a-b e^{-s x} ; \quad A^{+}(x, p)=-i p+a-b e^{-s x} .
$$

As we know 10, Morse potential is shape invariant. The relevant parameters of the model are $a_{0}=a$ and $a_{1} \equiv$ $f\left(a_{0}\right)=a-s$ respectively. Thus

$$
H_{+}(x, p ; a)+g(a)=H_{-}(x, p ; a-s)+g(a-s) ; \quad g(a)=a^{2},
$$

and consequently, the energy spectrum is

$$
E_{n}^{-}(a)=a^{2}-(a-n s)^{2} .
$$

Let's find the ground state Wigner function $P_{0}^{-}(x, p)$ using

$$
A(x, p) * P_{0}^{-}(x, p)=0 .
$$

Writing the $*$-products in 101 as Bopp's shifts, we obtain

$$
\left\{i\left(p-\frac{i}{2} \overrightarrow{\partial_{x}}\right)+a-b e^{-s\left(x+\frac{i}{2} \overrightarrow{\partial_{p}}\right)}\right\} P_{0}^{-}(x, p)=0,
$$

which becomes a mixed differential/finite-difference equation

$$
\begin{aligned}
(a+i p) P_{0}^{-}(x, p) & =b e^{-s x} P_{0}^{-}(x, p-i s / 2) \\
& -\frac{1}{2} \partial_{x} P_{0}^{-}(x, p) .
\end{aligned}
$$

The above equation mixes the partial derivative with respect to one variable with a shift with respect to the other variable. This hints to a well-known 26 differential/finite-difference formula for the modified Bessel functions $K_{\nu}(x)$

$$
2 \partial_{x} K_{\nu}(x)+K_{\nu-1}(x)+K_{1+\nu}(x)=0 .
$$

The exponential term in the r.h.s. of equation (103) suggests that $K_{\nu}(x)$ depends on $x$ via an exponential term. The shift in momentum variable suggests that $p$ appears in the index $\nu$ of $K_{\nu}(x)$. Therefore we substitute in 103 the following ansatz

$$
P_{0}^{-}(x, p)=C_{1} e^{-C_{2} x} K_{\alpha(p)}\left(C_{3} e^{-s x}\right),
$$

where the constants $C_{1}, C_{2}, C_{3}$, and the function $\alpha(p)$ are to be determined. Plugging 105 into 103 , after some algebra, we find that 103 is identically satisfied for

$$
C_{2}=2 a, \quad C_{3}=\frac{2 b}{s} ; \text { and } \alpha(p)=\frac{2 i p}{s} .
$$

for any value of $C_{1}$, which is constrained by the normalization of the Wigner function (28). We obtain $C_{1}=\frac{2}{\pi s}(2 b / a)^{2 a / s}$. Thus, the ground state Wigner function for the Morse oscillator is

$$
P_{0}^{-}(x, p ; a)=\frac{2}{\pi s}\left(\frac{2 b}{a}\right)^{2 a / s} e^{-2 a x} K_{2 i p / s}\left(\frac{2 b}{s} e^{-s x}\right) .
$$

Finding the next excited states Wigner functions is now straightforward using the shape invariance $(93)$. For example, let us find $P_{1}^{-}(x, p ; a)$

$$
\begin{aligned}
& P_{1}^{-}(x, p ; a)=A^{+}(x, p ; a) * \frac{P_{0}^{-}(x, p ; a-s)}{E_{1}^{-}\left(a_{0}\right)} * A(x, p ; a) \\
&=\left[a-i p-b e^{-s x}\right] * \frac{P_{0}^{-}(x, p ; a-s)}{a^{2}-(a-s)^{2}} *\left[a+i p-b e^{-s x}\right] \\
&=\left[a-i\left(p-\frac{i}{2} \overrightarrow{\partial_{x}}\right)-b e^{-s\left(x+\frac{i}{2} \overrightarrow{\partial_{p}}\right)}\right] \frac{P_{0}^{-}(x, p ; a-s)}{2 a s-s^{2}} \\
& {\left[a+i\left(p+\frac{i}{2} \overleftarrow{\partial_{x}}\right)-b e^{-s\left(x-\frac{i}{2} \overleftarrow{\partial_{p}}\right)}\right] . }
\end{aligned}
$$

After some algebra, we arrive to

$$
P_{1}^{-}(x, p ; a)=\alpha(x) K_{\nu}(y)-\beta(x)\left[K_{\nu-1}(y)+K_{\nu+1}(y)\right],
$$

where $\nu=\frac{2 i p}{s} ; y=\frac{2 b}{s} e^{-s x}$, and

$$
\begin{aligned}
& \alpha(x)=\frac{2^{\frac{2 a}{s}} e^{-2 a x}\left(\frac{b}{a-s}\right)^{-2+\frac{2 a}{s}}}{2 \pi(2 a-s) s^{2}}\left[4 b^{2}+e^{2 s x}(2 a-s)^{2}\right], \\
& \beta(x)=\frac{4^{a / s} b e^{(-2 a+s) x}\left(\frac{b}{a-s}\right)^{-2+\frac{2 a}{s}}}{\pi s^{2}} .
\end{aligned}
$$

In figure (2) we illustrate the Wigner function $P_{1}(x, p)$ for the Morse oscillator. The graph is cut on the vertical axis to emphasize the details near the origin.

\section{CONCLUSIONS}

We have shown that quantum mechanics can be expressed equivalently in the language of "normal" functions defined on the phase space, endowed with the *product. The Moyal bracket corresponds to the operator commutator, and one can use it as an heuristic tool for quantization. In the classical limit $(\hbar \rightarrow 0)$ the Moyal bracket becomes simply the Poisson bracket, and the *product, becomes the normal commutative product of functions. In this picture, quantum mechanics appears 


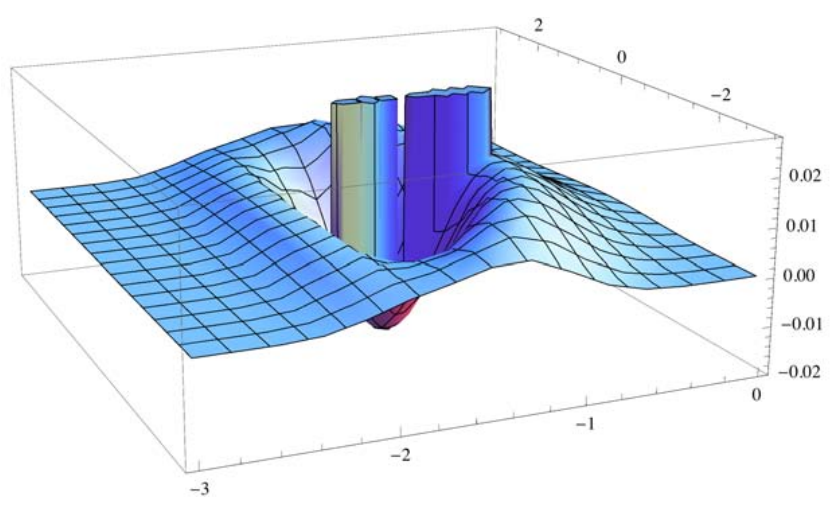

Figure 2: The Wigner function $P_{1}(x, p)$ for the Morse oscillator, for $a=5, b=1$, and $s=1$.

as a deformation of the classical mechanics, with the deformation parameter $\hbar$. Supersymmetric quantum mechanics induces additional relations between the Wigner functions, while shape invariance exposes a simple approach to recursively obtain the Wigner function of a system, starting from its ground state Wigner function, as exemplified for the Morse potential.

\section{Acknowledgements}

I would like to acknowledge a sabbatical leave and grant from Columbia College Chicago that made this work possible. I am grateful to Prof. Ennio Gozzi for hospitality extended to me during my visit to the Department of Physics at the University of Trieste -Miramare Campus (Italy), and for fruitful discussions. I would like to thank INFN for financial support (grant GE41), and CERN (Geneva, Switzerland) for hospitality.

[1] E. A. Akhundova, V. V. Dodonov, and V. I. Man'ko. Wigner functions of quadratic systems. Physica, 115 A:215-231, 1982.

[2] W. Arveson. Quantization and the uniqueness of invariant structures. Comm. Math. Phys., 89:77-102, 1983.

[3] G. Baker. Formulation of quantum mechanics based on the quasi-probability distribution induced on phase space. Phys. Rev., 109:2198-2206, 1958.

[4] M. S. Bartlett and J. E. Moyal. The exact transition probabilities of quantum-mechanical oscillators calculated by the phase-space method. Proc. Camb. Phil. Soc., 45:545-553, 1949.

[5] F. Bayen, M. Flato, C. Fronsdal, A. Lichnerowicz, and D. Sternheimer. Deformationtheory and quantization. i. deformations of symplectic structures. Ann. of Phys., 111:61-110, 1978.

[6] B. Belchev and M.A. Walton. Solving for the wigner functions of the morse potential in deformation quantization. J. Phys., A 43:225206-22518, 2010.
[7] F. A. Berezin. Feynman path integrals in a phase space. Sov. Phys. Usp, 23:763-788, 1980.

[8] F. Bopp. Werner Heisenberg und die Physik unserer Zeit. Vieweg, Braunschwieg, 1961.

[9] T. Chow. Mathematical Methods for Physicists - A Coincise Introduction. Cambridge Univ. Press, 2000.

[10] F. Cooper, A. Khare, and U. Sukhatme. Supersymmetry and quantum mechanics. Phys. Rep., 251:267-385, 1995.

[11] T. Curtright, D. Fairlie, and C. Zachos. Features of timeindependent wigner functions. Phys. Rev., D 58:02500202515, 1998.

[12] T. Curtright, T. Uematsu, and C. Zachos. Generating all wigner functions. J. Math. Phys., 42:2396-2415, 2001.

[13] M. de Wilde and P. Lecomte. Existence of star-products and of formal deformations of the poisson lie algebra of arbitrary symplectic manifolds. Lett. Math. Phys., 7:487496, 1983.

[14] P. A. M. Dirac. The relation of classical to quantum mechanics. In $2^{\text {nd }}$ Can. Math. Congress, Vancover 1949. U.Toronto Press, 1951.

[15] D. Fairlie. The formulation of quantum mechanics in terms of phase space functions. Proc. Camb. Phil. Soc., 60:581-586, 1964.

[16] M. Flato, A. Lichnerowicz, and A. Sternheimer. Deformations of poisson brackets, dirac brackets and applications. J. Math. Phys., 17:1754-1762, 1976.

[17] C. Fronsdal. Some ideas about quantization. Rep. Math. Phys., 15:111-145, 1978.

[18] A. Gangopadhyaya, J. V. Mallow, and C. Rasinariu. Supersymmetric Quantum Mechanics: An Introduction. World Scientific, 2011.

[19] E. Gozzi. Ground-state wave-function 'representation'. Phys. Lett., 129 B:432-436, 1983.

[20] E. Gozzi and M. Reuter. A proposal for a differential calculus in quantum mechanics. Int. J. Mod. Phys., A 9:2191-2227, 1994.

[21] H. J. Groenewold. On the principles of elementary quantum mechanics. Physica, 12:405-460, 1946.

[22] J. Hancock, M. A. Walton, and B. Wynder. Quantum mechanics another way. Eur. J. Phys., 25:525-534, 2004.

[23] M. Hillery, R. F. O'Connell, M. O. Scully, and E. P. Wigner. Distribution functions in physics: Fundamentals. Phys. Rep., 106:121-167, 1983.

[24] A. C. Hirshfeld and P. Henselder. Deformation quantization in the teaching of quantum mechanics. Am. J. Phys., 70:537-547, 2002.

[25] G. J. Iafrate, H. L. Grubin, and D. K. Ferry. The wigner distribution function. Phys. Lett., A 87:145-148, 1982.

[26] N. N. Lebedev. Special Functions and Their Applications. Dover, NY, 1972.

[27] H-W. Lee and M. O. Scully. Wigner phase-space description of a morse oscillator. J. Chem. Phys., 77:4604-4610, 1982.

[28] J. E. Moyal. Quantum mechanics as a statistical theory. Proc. Camb. Phil. Soc., 45:99-124, 1949.

[29] E. Schrödinger. A method of determining quantummechanical eigenvalues and eigenfunctions. Proc. R. Irish Acad., 46 A:9-16, 1940.

[30] J. Tosiek and M. Przanowski. Weyl-wigner-moyal formalism. i. operator ordering. Acta Phys. Pol., 26:1703-1716, 1995.

[31] L. van Hove. Sure certaines représentations unitaires. Proc. R. Acad. Sci. Belgium, 26:1-102, 1951.

[32] J. Vey. Déformation du crochet de poisson sur une variété 
symplectique. Comment. Math. Helv., 50:421-454, 1975.

[33] J. von Neumann. Die eindeutigkeit der schrödingerschen operatoren. Math. Ann., 104:570-578, 1931.

[34] H. Weyl. Quantenmechanik und gruppentheorie. $Z$. Phys., 46:1-46, 1927.

[35] E. Wigner. On the quantum correction for thermodynamic equilibrium. Phys. Rev., 40:749-759, 1932.
[36] E. P. Wigner. Perspectives in Quantum Theory. Dover, NY, 1979.

[37] E. Witten. Dynamical breaking of supersymmetry. Nucl. Phys., B 188:513-554, 1981.

[38] C. K. Zachos, D. B. Fairlie, and T. L. Curtright. Quantum Mechanics in Phase Space. World Scientific, 2005. 